SPICA Workshop, 04001 (2009)

DOI:10.1051/spica/200904001

(C) Owned by the authors, published by EDP Sciences, 2009

This is an Open Access article distributed under the terms of the Creative Commons Attribution-Noncommercial License, which permits unrestricted use, distribution, and reproduction in any noncommercial medium, provided the original work is properly cited.

\title{
SPITZER SPECTROSCOPY OF LIRGS AND ULIRGS
}

\author{
E. Sturm \\ Max-Planck-Institute for Extraterrestrial Physics, Giessenbachstr. 1, 85748 Garching, Germany
}

\section{ABSTRACT}

I summarize recent results from spectroscopic Spitzer studies of the evolution of (ultra-)luminous infrared galaxies, mainly from the QUEST and GOALS programmes. First, the mid-IR diagnostic toolbox is briefly introduced, with an emphasis on new insights on the calibration of zero points and bolometric corrections of these methods. Then various applications at low and high redshift are summarized, such as the AGN fraction in ULIRGs along a merger sequence, the star formation in QSO hosts, and the role of AGN at low and high z. These results illustrate some of the SPICA key science cases in the context of mid-IR spectroscopy of dusty galaxies at high redshifts.

Key words: Galaxies: formation - Galaxies: evolution Missions: SPICA

\section{INTRODUCTION}

Infrared bright Galaxies, such as (Ultra-)Luminous Infrared Galaxies (ULIRGs and LIRGs), with their huge infrared luminosities, correspond to an extremely active phase of dust enshrouded star formation and/or AGN activity. They become an increasingly significant population at high redshift, representing an important phase in the buildup of massive galaxy bulges and in the growth of their central super-massive black holes. To understand these processes, it is essential to separate the relative contributions of AGN and starburst activity to the infrared luminosity of LIRGs and ULIRGs.

The Spitzer Space Observatory has provided extremely valuable new insights into the evolution of dusty, infrared bright galaxies. A large number of spectra made it possible to fine tune and calibrate the infrared spectral toolbox, and to apply these methods in many different ways. These studies were limited though to the local universe or were restricted to $\mathrm{PAH}$ and continuum diagnostics only at higher redhifts out to $\mathrm{z} \approx 3$. It will be up to SPICA and its mid- and far-infrared spectrometers (MIRACLE and SAFARI) to carry out similar studies using the full mid-IR toolbox at redshifts around the peak of cosmic star formation and black hole evolution, in a consistent way and in much larger numbers. In the following section I will il- lustrate with a few examples how ISO and Spitzer paved the way to this key science case for SPICA.

\section{Calibrating the Infrared Toolbox}

Mid-IR fine structure line spectra contain tracers of low excitation gas (HII region) and high excitation gas (AGN narrow line and coronal region) as well as of partially ionized zones (AGN, shocks). Diagnostic diagrams equivalent to and extending the traditional optical ones can be used to classify and quantify activity in obscured objects (Genzel et al. 1998, Sturm et al. 2002, Verma et al. 2003, Lutz et al. 2003, Armus et al. 2007, Veilleux et al. 2009). Low resolution spectra covering the $5-40 \mu \mathrm{m}$ range can be decomposed into three components (Laurent et al. 2000). Starburst spectra are a mixture of a 'PDR' component dominated by the aromatic PAH emission features and a steeply rising 'HII' continuum, AGN can be identified on the basis of an additional flatter continuum. Absorption features from the strong silicate absorption to the more subtle signatures of ice and hydrocarbon absorption (Spoon et al. 2001, 2002, Tran et al. 2001) trace extremely obscured components, either compact starbursts as in the case of Arp 220 (Spoon et al. 2004) or deeply embedded AGN.

These methods have been pioneered by ISO, and refined and expanded by Spitzer. However, the results derived from different mid-IR tools show a significant scatter. Before the various tools can be applied to quantify the contributions from starbursts and AGN to the combined light of dusty galaxies, e.g. to study their evolution, it is necessary to adjust the toolbox such that different methods give consistent results. This requires two steps: first, the zero points of the relations have to be calibrated. For instance, what is the $[\mathrm{O} \mathrm{IV}] /[\mathrm{Ne} \mathrm{II}]$ ratio in pure AGN and in starbursts? Second, for the extrapolation from these mid-IR diagnostics to bolometric luminosities, i.e. to contributions to the entire light of the galaxy, bolometric corrections have to be established.

Veilleux et al. 2009 (V09 hereafter) use a large set of Spitzer IRS data from their QUEST programme (the Quasar and ULIRG Evolution Study) to estimate the fractional contribution of nuclear activity to the bolometric luminosity of the ULIRGs and PG QSOs in their sample (herafter called the AGN contribution for short). They use six different methods based on (1) the $[\mathrm{O}$ IV] $25.9 \mu \mathrm{m}$ 
$/[\mathrm{Ne} \mathrm{II}] 12.8 \mu \mathrm{m}$ ratio, $(2)$ the $[\mathrm{Ne} \mathrm{V}] 14.3 \mu \mathrm{m} /[\mathrm{Ne} \mathrm{II}] 12.8 \mu \mathrm{m}$ ratio, (3) the equivalent width of $\mathrm{PAH} 7.7 \mu \mathrm{m}$, (4) the PAH $(5.9-6.8 \mu \mathrm{m})$ to continuum $(5.1-6.8 \mu \mathrm{m})$ flux ratio combined with the continuum $(14-15 \mu \mathrm{m}) /(5.1-$ $5.8 \mu \mathrm{m}$ ) flux ratio, (5) the MIR blackbody to FIR flux ratio, and (6) the f30/f15 continuum flux ratio. An example, combining methods (1) and (2), is shown in figure 1 . Note that $[\mathrm{Ne} \mathrm{II}] 12.8 \mu \mathrm{m}$ and $[\mathrm{Ne} \mathrm{V}] 14.3 \mu \mathrm{m}$ can be easily substituted by the similarly strong lines of $[\mathrm{S} \mathrm{III}] 33.5 \mu \mathrm{m}$ and $[\mathrm{NeV}] 24.3 \mu \mathrm{m}$. With these modifications, which bring the three lines into a common wavelength range, the diagram in figure 1 can be directly applied to SPICA observations for sources at redshifts $\mathrm{z} \geq 0.2$.

V09 derive zero points and bolometric corrections for each method. They find good agreement, on average, between the 6 methods, once the zero point calibrations and bolometric corrections have been applied (figure 2). There remains, however, some scatter which can be attributed to uncertainties in the zero points, the bolometric correction, and other effects like differential extinction between different line emitting regions. The best use of this toolbox is certainly its application to galaxy samples, rather than single objects. We note in passing that the low spectral resolution diagnostics (PAH, continuum shape, color) are nicely confirmed by the more direct high resolution diagnostics.

\section{Applications at low And high Redshifts}

\subsection{ULIRG EVOLUTION (QUEST)}

Spitzer studies of ULIRGs, like the QUEST programme, have established that ULIRGs fall in one of three distinct AGN classes: (1) objects with small extinctions and large polycyclic aromatic hydrocarbon $(\mathrm{PAH})$ equivalent widths are highly starburst-dominated; (2) systems with large extinctions and modest $\mathrm{PAH}$ equivalent widths have larger AGN contributions, but still tend to be starburstdominated; and (3) ULIRGs with both small extinctions and small PAH equivalent widths host AGN that are at least as powerful as the starbursts. The AGN contributions in class 2 ULIRGs are more uncertain than in the other objects, and one cannot formally rule out the possibility that these objects represent a physically distinct type of ULIRGs. V09 found, when comparing the results of the AGN contribution to morphological tracers of the galaxy merger state (tidal tales, nuclear separation, etc.) and to the level of extinction (measured via the silicate absorption feature), that a morphological trend is seen along the sequence (1)-(2)-(3). Their results are in general agreement with the standard ULIRG-QSO evolution scenario and suggestive of a broad peak in extinction during the intermediate stages of merger evolution. However, the scatter in this sequence, including the presence of a significant number of AGN-dominated systems prior to coalescence and starburst-dominated but fully merged systems, implies that black hole accretion, in addition to depend-

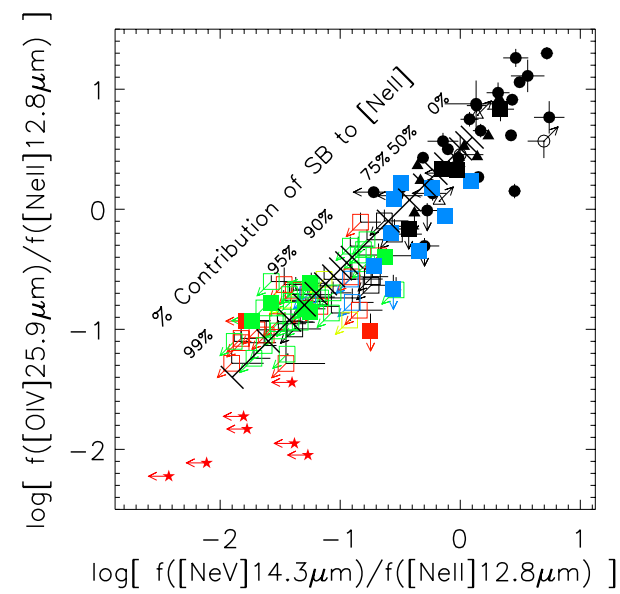

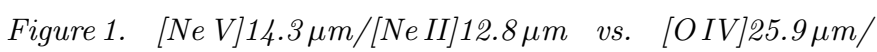
[Ne II]12.8 $\mu \mathrm{m}$ for ULIRGs and PG QSOs in the VO9 QUEST sample. Open symbols are upper limits. The colors of the symbols reflect the optical spectral types: red, green, blue, and black squares are H II-like, LINER, Seyfert 2, and Seyfert 1 ULIRGs, respectively. A clear positive correlation is observed, representing an excitation sequence anchored with the $H I I-$ like/LINER ULIRGs, moving up in excitation level to the Seyfert 2 and Seyfert 1 ULIRGs, and ending with the $P G$ QSOs. The solid diagonal line in these diagrams indicates constant [Ne V]/[O IV] and changing [Ne II]. This line may be interpreted as a mixing line if [Ne V] and [OIV] are only produced by an AGN and [NeII] by starburst activity. The tickmarks along the line indicate the percent contribution of the starburst to [Ne II]. The anchor point of this line has some systematic uncertainty due to variable AGN physical conditions. From V09.

ing on the merger phase, also has a strong chaotic/random component, as in local AGNs (multiple episodes of AGN dominance during late merger processes, pre-existing AGN, stochastic processes involved in AGN feeding only loosely connected to the large-scale merger process, etc.).

\subsection{QSO HOSTS}

To investigate the link between AGN activity and star formation and the extent to which they occur simultaneously, it is important to quantify the star formation activity in QSO hosts. Such measurements are made difficult, however, by the observational problems of detecting star formation tracers in the presence of extremely powerful AGN emission. In the MIR, the contrast between the emission from possibly dust-obscured star formation and from the central AGN is favourable, and established star formation tracers are available, as shown above. Schweitzer et al. (2006) have studied a sample of PG QSOs with Spitzer, and detected tracers of the star formation (PAH, lowexcitation $[\mathrm{Ne} I \mathrm{I}]$ ) in the host galaxies for many objects of 


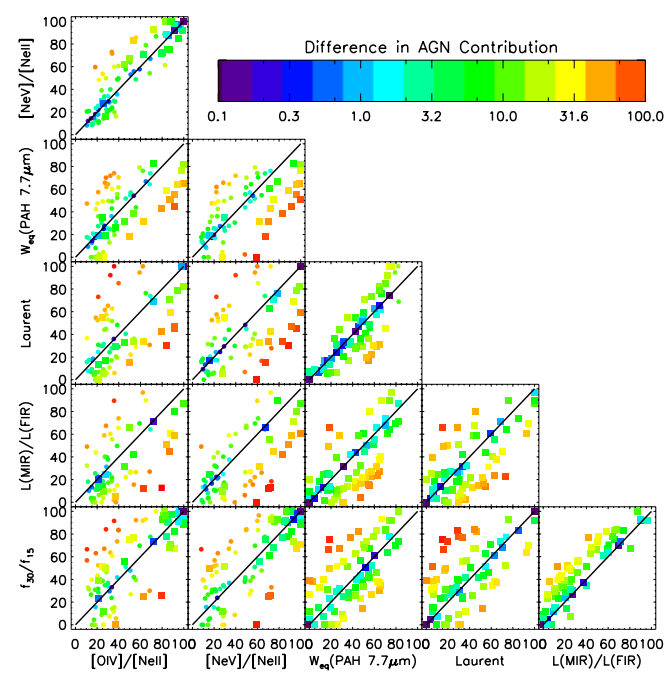

Figure 2. Comparison of AGN fractional contributions to the bolometric luminosities of ULIRGs and PG QSOs derived from 6 different methods (see text). Squares are actual AGN fractional contributions, while small circles are upper limits on the AGN fractional contributions derived from the fine structure line ratios. The colors represent deviations $(x-y)$ from the line of equality, on a logarithmic scale. Good agreement is seen on average between the various methods. From V09.

their sample. They find strong correlations of far-infrared continuum and these starburst related quantities. They conclude that the typical QSO in their sample has at least $30 \%$ but likely most of the far-infrared luminosity arising from star formation, with a tendency for larger star formation contribution at the largest FIR luminosities. There are also correlations between AGN tracers and starburst tracers. The common scaling of AGN and starburst luminosities (and fluxes) is evidence for a starburst-AGN connection in luminous AGN.

Lutz et al. 2008 expanded this study to higher redshifts. They obtained Spitzer rest-frame mid-infrared spectra of twelve $\mathrm{z} \approx 2 \mathrm{~mm}$-bright type 1 QSOs, selected from unlensed and lensed QSO samples. On top of the AGN continuum, they detect PAH emission from luminous star formation in nine objects individually as well as in the composite spectrum for the full sample. PAH luminosity and rest frame far-infrared luminosity correlate and extend the similar correlation derived for the Schweitzer et al. sample of lower luminosity local QSOs, see figure 3. This provides strong evidence for intense star formation in the hosts of these mm-bright QSOs, sometimes exceeding $1000 \mathrm{M} \odot / \mathrm{yr}$.

\subsection{The role of AGN neAr AND far (GOALS)}

Studies of galaxy evolution are often performed on the basis of galaxy counts, i.e. on the number distribution of galaxies as a function of their luminosity and redshift. For

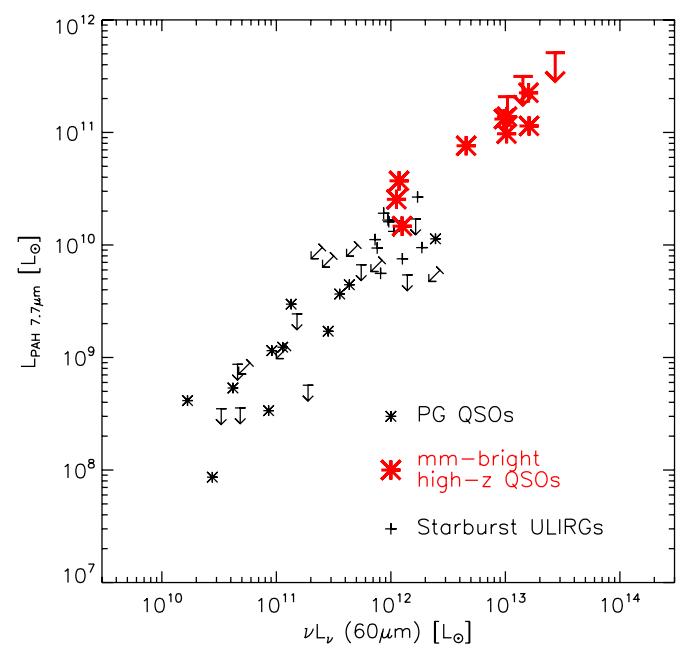

Figure 3. Relation of the PAH $7.7 \mu m$ luminosity and farinfrared continuum luminosities for the sample of high-z QSOs from Lutz et al. (2008) and for local comparison objects. $P G Q S O s$ as well as the comparison sample of starbursting ULIRGs are from the studies of Schweitzer et al. (2006) and Netzer et al. (2007). For lensed high-z objects, luminosities have been corrected for the magnifications. From Lutz et al. (2008).

modeling these number counts, often the so-called backward evolution approaches are invoked. These methods take the observed, present day $(\mathrm{z}=0)$ luminosity function and evolve it in luminosity and/or density out to higher redshifts assuming some parametrization of the evolution (e.g. Franceschini at al. 1988; Pearson \& Rowan-Robinson 1996; Xu et al. 2001; Franceschini et al. 2001; RowanRobinson 2001; Lagache et al. 2003). The "classical" backward evolution model starts from different populations of galaxies, typically cirrus, starburst, active galactic nuclei (AGN) and ultraluminous infrared galaxies (ULIRGs), or a subset of those. Each population is assigned a spectral energy distribution (SED) and local luminosity function, and evolves independently, assuming it has a proper cosmic evolution rate.

Backward evolution models based on single SEDs or a simple SED family approach have been quite successful in fitting the number counts from the IRAS and ISO missions. The number and quality of observed constraints is increasing, however, and already the first Spitzer results have led to on-the-spot modifications (c.f. the modified SEDs adopted by Lagache et al. 2004) that may either genuinely represent improved knowledge, or reflect limitations of the simple assumptions made previously. Furthermore, questions gaining increased importance, like the co-existence of AGN and star-formation in infrared galaxies, cannot be addressed by this generation of models, not even in the simple sense of fitting existing data and extrapolating to new observations. The next step to be taken was the development of a backward evolution model that 
considers realistic spreads in far-infrared SEDs, in AGN contributions at different luminosities, and their possible evolution with redshift. Valiante et al. (2009) have started such an effort, based on Spitzer archive data of LIRGS and ULIRGs, mainly from the QUEST and GOALS programmes. GOALS, the Great Observatory All-sky LIRG Survey, is a multi-wavelength imaging and spectroscopy survey of 200 local Luminous Infrared Galaxies, combining data from NASAs Spitzer, Chandra, Hubble and GALEX observatories (see the overview paper by Armus et al. 2009). In order to be useful for backward modelling projects as outlined above, a local calibration of the distribution of AGN contributions at a given infrared luminosity has to span the highest luminosities down to at least the low end of the LIRG regime $\left(10^{11} \mathrm{~L} \odot\right)$. What is needed is a quantification of AGN content for an unbiased far-IR selected sample reaching down to $10^{11} \mathrm{~L} \odot$. Historically, many of the references cited above have focussed on the ULIRG $(\geq$ $\left.10^{12} \mathrm{~L} \odot\right)$ regime and even its upper end where the AGN contribution is highest, while studies at lower luminosities mostly focussed on individual interesting objects. With the GOALS project such a large sample of LIRGs became available now.

Valiante et al. found a correlation between the AGN contribution and the total far-IR luminosity of a galaxy (figure 4). This was already known for ULIRGs but had not been systematically quantified, and it had not been demonstrated for LIRGs before. It is also necessary to check if and how the relation found between AGN contribution and $\mathrm{L}_{T I R}$ evolves with redshift. Previous studies already showed that, even if in the local universe ULIRGs tend to be AGN-dominated at $\mathrm{L}_{T I R} \geq 10^{12.5} \mathrm{~L} \odot($ Lutz et al. 1998; Veilleux et al. 1999; Tran et al. 2001), this trend does not extend to higher redshifts. For example, submillimeter galaxies that, with their typical infrared luminosity of $10^{13} \mathrm{~L} \odot$, are among the most luminous objects known, are mainly starburst dominated. Mid-IR spectra in fact show that the contribution to the total luminosity due to AGN continuum is small in most of the sources observed with Spitzer IRS at high redshifts (Lutz et al. 2005a; Menendez-Delmestre et al. 2007; Valiante et al. 2007; Pope et al. 2008). These results are consistent with X-ray observations of the same population (Alexander et al. 2005). Valiante et al. have confirmed now that the relation does not hold any more at high redshifts. The most luminous high redshift infrared galaxies, $\mathrm{L}_{T I R} \geq 10^{13} \mathrm{~L} \odot$, show a small contribution from AGN, being mainly starburst powered. This result, however, is tentative and based on a very limited data set of Spitzer spectra of high redhsift AGN. SPICA will obviously make a very important contribution in this field.

\section{IMPLICATIONS FOR SPICA}

Recent work has shown how the IRAC color-color diagram and the MIPS 24 to $8 \mu \mathrm{m}$ color can be used to identify

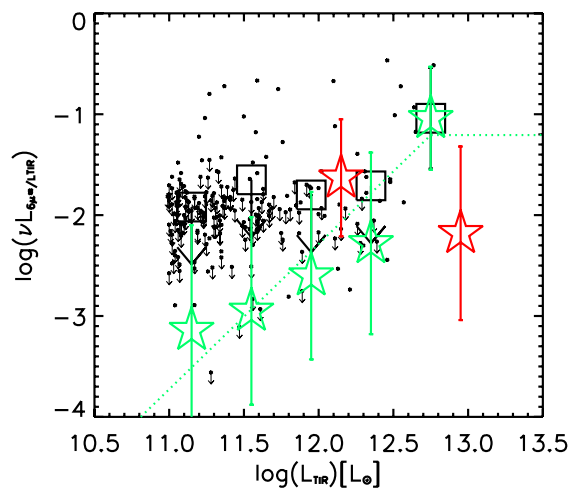

Figure 4. AGN contribution, parametrized as the ratio of the $A G N$ continuum luminosity at $6 \mu \mathrm{m}$ vs. $L_{T I R}$, as a function of $L_{T I R}$. Dots and small arrows represent measurements and upper limits of all the galaxies in the local sample. The large open squares are the means of the detections in bins of luminosity. Due to the large number of non-detections, they represent only an upper-limit on the true AGN contribution for almost all the bins. Valiante et al. have therefore applied monte carlo simulations adopting gaussian distributions in order to reproduce detection rates, means and sigma of the detections from the measurements. The green stars and the associated bars show means and sigma of these gaussian distributions. The best fit is shown as a dotted green line: the relation is assumed to be flat at high LTIR. The red stars and their bars show means and sigma of the gaussian distributions adopted to reproduce measurements of the high redshifts sample. The relation found for the local galaxies no longer holds. From Valiante et al. 2009.

AGN-dominated sources (Lacy et al. 2004; Sajina et al. 2005; Stern at al. 2005; Yan et al. 2004; Brand et al. 2006). However, a photometric study of this type is subject to several caveats. Many broad emission- and absorption-line features are known to be present in the mid-IR spectrum of ULIRGs (e.g. Houck et al. 2005; Yan et al. 2005), and this may affect as function of redshift. For example, at 1.1 $\leq \mathrm{z} \leq 1.7$, the $24 \mu \mathrm{m}$ observed emission can be strongly attenuated by the silicate absorption feature at $9.7 \mu \mathrm{m}$. It is also possible that an AGN could be heavily embedded in large amounts of cooler dust and could remain undetected in the $8 \mu \mathrm{m}$ band even though it dominates the bolometric infrared emission. In practice: on one hand, the galaxies, which in Brand et al. (2006) are classified as AGN-dominated, do host an AGN, but it is not necessarily the dominant source powering the infrared emission; on the other hand, objects that are known to be dominated by AGN in the mid-IR, like NGC1068, do not present the color that is considered typical for AGN-dominated sources in the Brand et al. (2006) study. Obtaining mid-IR spectroscopy of complete unbiased mid-IR selected samples of infrared sources will be important in putting more constraints on the AGN contribution to the total infrared 
emission and in characterization of that population. This will be one of the prime tasks for SPICA.

The examples shown above (plus many others not mentioned here) provide all the necessary ingredients (e.g. template spectra covering a wide range of galaxy properties and sub-classes, calibrated mid-IR diagnostic tools with proper zero points, etc.) for planning and interpreting SPICA observations of the dusty, high redshift universe at the peak epoch of cosmic star formation and black hole evolution.

\section{REFERENCES}

Alexander, D.M., Bauer, F.E., Chapman, S.C., Smail, I. Blain, A.W., Brandt, W.N., Ivison, R.J. 2005, ApJ, 632, 736

Armus, L., et al. 2007, ApJ, 656, 148

Armus, L., et al. 2009, PASP, 121, 559

Brand, K., et al. 2006, ApJ, 644, 143

Franceschini, A., Danese, L., de Zotti, G., Xu, C. 1988, MNRAS, 233, 175

Franceschini, A., Aussel, H., Cesarsky, C.J., Elbaz, D., Fadda, D. 2001, A\&A, 378, 1

Genzel, R., et al. 1998, ApJ, 498, 579

Houck, J.R., et al. 2005, ApJL, 622, L105

Lacy, M., et al. 2004, ApJs, 154, 166

Lagache, G., Dole, H., Puget, J.L. 2003, MNRAS, 338, 555

Lagache, G., et al. 2004, ApJs, 154, 112

Laurent, O., Mirabel, I.F., Charmandaris, V., Gallais, P., Madden, S.C., Sauvage, M., Vigroux, L., Cesarsky, C. 2000, A\&A, 359, 887

Lutz, D., Spoon, H.W.W, Rigopoulou, D., Moorwood, A.F.M., Genzel, R. 1998, ApJl, 505, L103

Lutz, D., Sturm, E., Genzel, R., Spoon, H. W. W., Moorwood, A. F. M., Netzer, H., \& Sternberg, A. 2003, A\&A, 409, 867

Lutz, D., Valiante, E., Sturm, E., Genzel, R., Tacconi, L.J., Lehnert, M.D., Sternberg, A., Baker, A.J. 2005, ApJ, 625, L83

Lutz, D., et al. 2008, ApJ, 684, 853

Menéndez-Delmestre, K., et al. 2007, ApJ, 655, 65L

Pearson, C.P. \& Rowan-Robinson, M. 1996, MNRAS, 283, 174

Pope, A., et al. 2008, ApJ, 675, 1171

Rowan-Robinson, M. 2001, ApJ, 549, 745

Sajina, A., Lacy, M., Scott, D. 2005, ApJ, 621, 256

Schweitzer, M., et al. 2006, ApJ, 649, 79

Spoon, H. W. W., Keane, J. V., Tielens, A. G. G. M., Lutz, D., \& Moorwood, A. F. M. 2001, A\&A, 365, L353

Spoon, H. W. W., Keane, J. V., Tielens, A. G. G. M., Lutz, D., Moorwood, A. F. M., \& Laurent, O. 2002, A\&A, 385, 1022

Spoon, H. W. W., et al. 2004, ApJs, 154, 184

Stern, D., et al. 2005, ApJ, 631, 163

Sturm, E., Lutz, D., Verma, A., Netzer, H., Sternberg, A., Moorwood, A.F.M., Oliva, E., Genzel, R. 2002, A\&A,
393,821

Tran, Q.D., et al. 2001, ApJ, 552, 527

Valiante, E., Lutz, D., Sturm, E., Genzel, R., Tacconi, L.J., Lehnert, A., Baker, A.J. 2007, ApJ, 660, 1060

Valiante, E., Lutz, D., Sturm, E., Genzel, R., \& Chapin, E. L. 2009, ApJ, 701, 1814

Veilleux, S., Kim, D.-C., Sanders, D.B. 1999, ApJ, 522, 113

Veilleux, S., et al. 2009, ApJs, 182, 628 (V09)

Verma, A., Lutz, D., Sturm, E., Sternberg, A., Genzel, R., \& Vacca, W. 2003, A\&A, 403, 829

Xu, C., Lonsdale, C.J., Shupe, D.L., O'Linger, J., Masci, F. 2001, ApJ, 562, 179

Yan L. et al. 2004, ApJs, 154, 60

Yan, L., et al. 2005, ApJ, 628, 604 